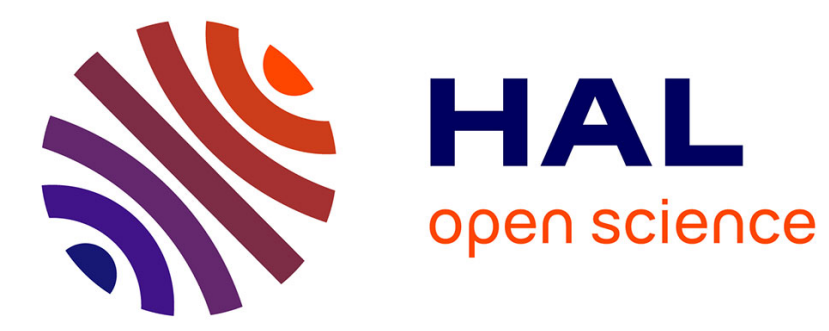

\title{
Medical image registration using Edgeworth-based approximation of Mutual Information
}

\author{
Mathieu Rubeaux, Jean-Claude Nunes, Laurent Albera, Mireille Garreau
}

\section{To cite this version:}

Mathieu Rubeaux, Jean-Claude Nunes, Laurent Albera, Mireille Garreau. Medical image registration using Edgeworth-based approximation of Mutual Information. Innovation and Research in BioMedical engineering, 2014, 35 (3), pp.139-148. hal-00914411v2

HAL Id: hal-00914411

https://hal.science/hal-00914411v2

Submitted on 13 Dec 2013

HAL is a multi-disciplinary open access archive for the deposit and dissemination of scientific research documents, whether they are published or not. The documents may come from teaching and research institutions in France or abroad, or from public or private research centers.
L'archive ouverte pluridisciplinaire HAL, est destinée au dépôt et à la diffusion de documents scientifiques de niveau recherche, publiés ou non, émanant des établissements d'enseignement et de recherche français ou étrangers, des laboratoires publics ou privés. 


\title{
Medical image registration using Edgeworth-based approximation of Mutual Information
}

\author{
Mathieu Rubeaux, Jean-Claude Nunes, Laurent Albera, and Mireille Garreau
}

\begin{abstract}
We propose a new similarity measure for iconic medical image registration, an Edgeworth-based third order approximation of Mutual Information (MI) and named 3-EMI. Contrary to classical Edgeworth-based MI approximations, such as those proposed for independent component analysis, the 3-EMI measure is able to deal with potentially correlated variables. The performance of 3-EMI is then evaluated and compared with the Gaussian and B-Spline kernel-based estimates of MI, and the validation is leaded in three steps. First, we compare the intrinsic behavior of the measures as a function of the number of samples and the variance of an additive Gaussian noise. Then, they are evaluated in the context of multimodal rigid registration, using the RIRE data. We finally validate the use of our measure in the context of thoracic monomodal non-rigid registration, using the database proposed during the MICCAI EMPIRE10 challenge. The results show the wide range of clinical applications for which our measure can perform, including non-rigid registration which remains a challenging problem. They also demonstrate that 3-EMI outperforms classical estimates of MI for a low number of samples or a strong additive Gaussian noise. More generally, our measure gives competitive registration results, with a much lower numerical complexity compared to classical estimators such as the reference B-Spline kernel estimator, which makes 3-EMI a good candidate for fast and accurate registration tasks.
\end{abstract}

Index Terms - cumulants, Edgeworth expansion, medical image registration, mutual information.

\section{INTRODUCTION}

$\mathbf{R}$ EGISTRATION aims at identifying the geometric transformation between two images or 3D volumes which minimizes a cost or objective function. Many automatic image registration methods have been proposed in the literature (see [1], [2] for a bibliographical survey). In the context of iconic multidimensional non-rigid and multimodal medical image registration, the techniques based on Mutual Information (MI) [3], [4] are the most common [5], [6]. In practice, MI cannot be computed exactly and has to be derived from an estimation of marginal and joint Probability Density Functions (PDF's). There are essentially three different techniques to estimate these PDF's: histogram-based estimators [7], kernel-based estimators [8], [9] and parametric methods. Parametric methods are clearly not appropriate to the context of medical image registration due to the statistical complexity of the processed images. Histogram-based and kernel-based estimators are more attractive due to their simplicity. However, they suffer from variance, bias caused by i) the finite number of observations, ii) quantization and iii) the finite histogram. Moreover, the histogram binning is usually time-consuming.

In order to overcome these drawbacks, a new similarity measure is proposed in this paper, which is an approximation of MI based on a third order Edgeworth expansion of marginal and joint PDF's. This idea has been introduced two decades ago in the context of Independent Component Analysis (ICA) [10]. Nevertheless, the different approximations derived for ICA can't be used for our registration

M. Rubeaux, J. C. Nunes, L. Albera and M. Garreau are with INSERM, UMR 1099, Rennes, F-35000, France and Université de Rennes 1, LTSI, F-35000, France, L. Albera is also with the Centre INRIA RennesBretagne Atlantique, Rennes 35042, France (e-mails: mrubeaux@gmail.com, jean-claude.nunes@univ-rennes1.fr, mireille.garreau@univ-rennes1.fr). task, since they assume a decorrelation of the involved random variables. For that reason, we developped novel approximations, leading to the novel 3-EMI metric which is compared to Gaussian [3] and B-Spline [11], [12] kernel-based estimations of MI (named $\mathrm{MI}_{\mathrm{G}}$ and $\mathrm{MI}_{\mathrm{BS}}$, respectively, in the following). 3-EMI metric has been briefly described in [13]. We give here an extensive validation study, as well as the mathematical details needed to understand this approach. In section II, we explain our method and the mathematical developments that were led to obtain this new measure. In section III, we detail the global registration scheme used during the experiments, giving the choices we made regarding the other components of this scheme. Section IV shows the results we obtained. First, we measure the intrinsic behavior of the three metrics (3-EMI, $\mathrm{MI}_{\mathrm{G}}$ and $\mathrm{MI}_{\mathrm{BS}}$ ) as a function of i)the number of samples and ii)the variance of an additive Gaussian noise. Then, we evaluate their performance on two different medical image registration problems. For that purpose, we use the RIRE database [14], which gives gold-standard registration results in the context of multimodal rigid registration. Next, we apply our measure and $\mathrm{MI}_{\mathrm{BS}}$ to the task of non-rigid registration of lungs. The database used [15] [27] gives the opportunity to estimate the quality of non-rigid registration based on two scores. Finally, we analyse the numerical complexity of 3- EMI. The 3EMI measure appears more robust with respect to a low number of samples or a strong additive Gaussian noise than classical measures. The results also show the wide range of clinical applications for which our measure can perform. They demonstrate that 3-EMI is particularly effective for rigid and non-rigid registration tasks: it outperforms classical estimates of MI, giving the best compromise between performance and numerical complexity.

\section{TOWARD THE 3-EMI SIMILARITY MEASURE}

Let $I_{F}(\boldsymbol{\nu})$ be a random variable modeling a floating image we want to register to a reference image $I_{R}(\boldsymbol{\nu})$, where $\boldsymbol{\nu}$ is a random vector over coordinate locations in the model. To build a registration procedure, one has to define the basic components of the registration framework: i) the feature space (the characteristics of the images taken into account), ii) the similarity measure used to compare these characteristics, iii) the type of geometrical transformation we consider, and iv) the chosen optimization method. In our context, the feature space is spanned by the pixel intensities of both images to register. While points iii) and iv) will be dealt with in section III, now let focus on the design of a new similarity measure. For the sake of convenience, let's define a vector $\boldsymbol{x}$ of two random variables $x^{(1)}$ and $x^{(2)}$, which will denote $I_{F}(\boldsymbol{\nu})$ and $I_{R}(\boldsymbol{\nu})$, respectively.

\section{A. MI: a statistical similarity measure}

Shannon entropy [16] $H(x)$ of a random variable $x$ is given by:

$$
H(x)=-\int_{\mathbb{R}} p_{x}(u) \log \left(p_{x}(u)\right) d u
$$

where $p_{x}$ is the marginal PDF of $x$. The joint entropy $H(\boldsymbol{x})$, measuring the dispersion of the joint PDF, $p_{\boldsymbol{x}}$, of the vector $\boldsymbol{x}$ is 
defined by:

$$
H(\boldsymbol{x})=-\oint_{\mathbb{R}^{2}} p_{\boldsymbol{x}}(\boldsymbol{u}) \log \left(p_{\boldsymbol{x}}(\boldsymbol{u})\right) d \boldsymbol{u}
$$

In information theory, MI gives a measure of the statistical dependence between two random variables. It can be expressed as a function of the marginal and joint entropies of $x^{(1)}$ and $x^{(2)}$ :

$$
M I(\boldsymbol{x})=H\left(x^{(1)}\right)+H\left(x^{(2)}\right)-H(\boldsymbol{x})
$$

As shown in equation (3), the computation of MI requires in practice the estimation of the marginal and joint PDF's of $\boldsymbol{x}$. The estimation method that is commonly used consists in building marginal and joint histograms [17]. For the marginal PDF, one has to count the occurrence of values at points within an image. For the joint PDF, given several realizations of vector $\boldsymbol{x}$ provided by both images to register, a joint histogram is built that tells us how often pairs of values occur together. Moreover, these histograms can be smoothed using Gaussian [3] or B-Spline [11], [12] kernels. In our experiments, we will compare these two kernel-based MI measures to our 3-EMI metric.

\section{B. EMI: Edgeworth-based approximation of MI}

Edgeworth expansion aims at approximating a PDF from its cumulants and Hermite polynomials. The expansion is computed around the closer normal distribution, the terms of the expansion giving the distance to normality. It has been intensively studied in signal processing, and more particularly in ICA [10] in order to approximate multivariate differential entropies [18]. Nevertheless, the latter approximations cannot be applied to the registration task. Indeed, such approximations require to prewhiten the data in order to considerably simplify their computation. However, in a registration scheme, this prewhitening step doesn't make sense because the aim is to find the perfect match between random variables, i.e. a total statistical dependence. So new approximations had to be developped without any decorrelation assumption.

Given a zero-mean bidimensional random vector $\boldsymbol{x}=\left[x^{(1)}, x^{(2)}\right]$, second and third order cumulants of $\boldsymbol{x}$ are defined by:

$$
\begin{aligned}
\kappa^{i_{1}, i_{2}}(\boldsymbol{x}) & =\mathrm{E}\left[x^{\left(i_{1}\right)} x^{\left(i_{2}\right)}\right] \\
\kappa^{i_{1}, i_{2}, i_{3}}(\boldsymbol{x}) & =\mathrm{E}\left[x^{\left(i_{1}\right)} x^{\left(i_{2}\right)} x^{\left(i_{3}\right)}\right]
\end{aligned}
$$

where $E[$.$] denotes the mathematical expectation operator. For in-$ stance, the third order marginal cumulant of the zero-mean random variable $x^{(i)}$ is given by $\kappa^{i, i, i}(\boldsymbol{x})=\mathrm{E}\left[\left(x^{(i)}\right)^{3}\right]$. Regarding Hermite polynomials, definitions and useful properties are recalled in appendix A.

We assume hereafter that both random variables of $\boldsymbol{x}$ have zeromean and unit-variance. In our context, it means that our images have been centered and standardized: this is far less restrictive than the prewhitening step discussed above, and it's a common preprocessing step in image registration. The univariate expansion up to order 3 of the marginal PDF of $x^{(i)}$ is given by [19]:

$$
p_{x^{(i)}}\left(u^{(i)}\right) \approx \phi_{x^{(i)}}\left(u^{(i)}\right)\left(1+\frac{1}{3 !} \kappa^{i, i, i}(\boldsymbol{x}) H_{3}\left(u^{(i)}\right)\right)
$$

where $\phi_{x^{(i)}}, H_{3}$ are the marginal standard normal distribution and the third order Hermite polynomial, given by (16) and (17), respectively. The bivariate expansion up to order 3 of the joint PDF $p_{x}$ is defined by [19]:

$$
p_{\boldsymbol{x}}(\boldsymbol{u}) \approx \phi_{\boldsymbol{x}}(\boldsymbol{u})\left(1+B_{\boldsymbol{x}}(\boldsymbol{u})\right)
$$

where $\phi_{\boldsymbol{x}}$ is the bivariate standard normal distribution given by (24) and where $B_{\boldsymbol{x}}$ is defined by:

$$
\begin{aligned}
B_{\boldsymbol{x}}(\boldsymbol{u}) & =\frac{1}{3 !}\left(\kappa^{1,1,1} H_{1,1,1}(\boldsymbol{u})+3 \kappa^{1,1,2} H_{1,1,2}(\boldsymbol{u})\right. \\
& \left.+3 \kappa^{1,2,2} H_{1,2,2}(\boldsymbol{u})+\kappa^{2,2,2} H_{2,2,2}(\boldsymbol{u})\right)
\end{aligned}
$$

with $H_{i_{1}, i_{2}, i_{3}}$ the third order bivariate Hermite polynomial defined by (21-22).

Using these expansions, we can approximate the marginal (1) and joint (2) entropies. These approximations are given in the following. The detailed mathematical developments used for constructing the joint entropy are proposed in appendix $\mathrm{C}$.

It is noteworthy that an approximation of marginal entropy is given in [20]. However, the approximation uses a second order Taylor expansion of $\log (1+x)$, which leads to intractable mathematical expressions when an approximation of joint entropy is expected. In the developments we conducted, we prefered to use a second order Taylor expansion of $(1+x) \log (1+x)$, which has already been used [10], [18] for negentropy estimation, and permits as well joint entropy approximation, as exposed in appendix C. We thus also developed novel approximations of marginal entropies, following the same formalism as for joint entropy, which won't be developed in detail. This leads to a slightly different approximation of the marginal entropy, compared to the one in [20], given, at third order, by:

$$
H_{3-E M I}\left(x^{(i)}\right)=\frac{1}{2} \log (2 \pi \mathrm{e})-\frac{1}{2} \frac{\kappa^{i, i, i}(\boldsymbol{x})^{2}}{3 !}
$$

The approximation of joint entropy for potentially correlated random variables is given by:

$$
\begin{aligned}
& H_{3-E M I}(\boldsymbol{x})=1+\log (2 \pi)+0.5 \log \left(1-\rho^{2}\right) \\
& -\frac{1}{12\left(1-\rho^{2}\right)^{3}}\left(\kappa^{1,1,1^{2}}+\kappa^{2,2,2^{2}}+3 \kappa^{1,1,2^{2}}+3 \kappa^{1,2,2^{2}}\right. \\
& -6 \rho\left(\kappa^{1,1,1} \kappa^{1,1,2}+\kappa^{1,2,2} \kappa^{2,2,2}+2 \kappa^{1,1,2} \kappa^{1,2,2}\right) \\
& +6 \rho^{2}\left(\kappa^{1,1,2^{2}}+\kappa^{1,2,2^{2}}+\kappa^{1,1,1} \kappa^{1,2,2}+\kappa^{1,1,2} \kappa^{2,2,2}\right) \\
& \left.-2 \rho^{3}\left(\kappa^{1,1,1} \kappa^{2,2,2}+3 \kappa^{1,1,2} \kappa^{1,2,2}\right)\right)
\end{aligned}
$$

where $\rho=\kappa^{1,2}(\boldsymbol{x})$ is the covariance between $x^{(1)}$ and $x^{(2)}$ and where $\kappa^{i, i, i}$ denotes $\kappa^{i, i, i}(\boldsymbol{x})$.

With formulas (8) and (9), the MI expression (3) can be approximated. These formulas are mainly composed of multivariate cumulants. In practice, cumulants require to be estimated from several realizations of the involved random variables. More particularly, let $\left\{x^{(i)}[\ell]\right\}_{1 \leq \ell \leq L}$ be a set of $L$ independent realizations of the zeromean random variable $x^{(i)}$, we get:

$$
\begin{aligned}
& \frac{1}{L} \sum_{\ell=1}^{L} x^{\left(i_{1}\right)}[\ell] x^{\left(i_{2}\right)}[\ell] \underset{L \rightarrow+\infty}{\longrightarrow} \kappa^{i_{1}, i_{2}}(\boldsymbol{x}) \\
& \frac{1}{L} \sum_{\ell=1}^{L} x^{\left(i_{1}\right)}[\ell] x^{\left(i_{2}\right)}[\ell] x^{\left(i_{3}\right)}[\ell] \underset{L \rightarrow+\infty}{\longrightarrow} \kappa^{i_{1}, i_{2}, i_{3}}(\boldsymbol{x})
\end{aligned}
$$

\section{Numerical complexity analysis}

The aim of this section is to give the cost per iteration of the proposed similarity measure, in comparison with the numerical 
complexity of the classical $M I_{B S}$ measure. Numerical complexity measurement aims at giving a fair comparison between different algorithms. We prefer it to the CPU time, because it gives a computational cost which is independent from the computer used. It consists in counting the number of multiplications and additions required to compute the two similarity measures. We found out that our measure has a cost per iteration of $9 \mathrm{~L}+44+3 \log$, where $\mathrm{L}$ is the number of image samples taken into account in the approximation. We chose to keep log, the cost of a log function call, appart because it can slightly vary regarding the precision expected. In this calculation, the more important cost comes from the cumulants: according to (10), a third order cumulant has a $2 \mathrm{~L}+1$ cost. Thus, we can consider that our metric has a $O(\mathrm{~L})$ cost per iteration, since L remains large in practice compared to the other terms. Regarding the Parzen estimator using a B-Spline kernel, which will be shown in the following more efficient than that using a Gaussian kernel, the cost per iteration we found, according to formulae given in [11], [12] is $\mathrm{N}_{b}^{2}(5+\log +4 \mathrm{~L})$, where $\mathrm{N}_{b}$ is the number of histogram bins of the reference and floating image. We found a similar result in the literature [23], with a $O\left(\mathrm{LN}_{b}{ }^{2}\right)$ cost per iteration. In our experiments, we fixed $\mathrm{N}_{b}$ to 32 , which is a good compromise between speed and accuracy and is a recommended value.

So the main advantage of our new 3-EMI approximation is clearly brought to light here. Indeed, the computational cost per iteration of our 3-EMI approximation is much lighter than standard $\mathrm{MI}_{\mathrm{BS}}$ approximation, due to the fact that we don't need to build a joint histogram to compute our measure.

\section{REGISTRATION PROCEDURE SET-UP}

We defined above the 3-EMI similarity measure that will be compared to the classical $\mathrm{MI}_{\mathrm{G}}$ [3] and $\mathrm{MI}_{\mathrm{BS}}$ [11], [12] estimates of MI. We now have to set-up a global registration procedure to test the performances of these measures. We thus need to define the deformation model as well as an optimization procedure.

\section{A. Motion model}

For our purpose, three main types of transformations have to be considered. In section IV-B, we evaluate the quality of 3D multimodal rigid registration, so a rigid transformation modeled with 3 Euler angles and 3 translation parameters will be sufficient. In section IV-C, however, we perform non-rigid registration, and we will consider both an affine transformation model composed of 15 parameters in three dimensions, which will serve as a preprocessing step for the nonrigid registration which will use a Free-Form Deformation (FFD) model based on B-Splines [24]. B-Splines are very popular in medical image registration due to their pleasant properties: compacity of their support, separability in each dimension, derivability and a relative straightforward implementation. But they are above all a powerful tool for modeling deformable objects. The FFD model warps an image by moving an underlying set of control points distributed over a regular grid. The displacement of a point $\nu$ of the image can be written as a linear combination of B-Spline functions $\beta^{(k)}$, weighted by the parameters $\boldsymbol{\xi}^{(k)}$ in the neighborhood $\mathcal{K}(\boldsymbol{\nu})$ of this point:

$$
\phi(\boldsymbol{\nu}, \boldsymbol{\xi})=\sum_{k \in \mathcal{K}(\boldsymbol{\nu})} \boldsymbol{\xi}^{(k)} \beta^{(k)}(\boldsymbol{\nu})
$$

The non-rigid transformation linking $I_{R}(\boldsymbol{\nu})$ and $I_{F}(\boldsymbol{\nu})$ can then be written as:

$$
I_{R}(\boldsymbol{\nu})=I_{F}(\phi(\boldsymbol{\nu}, \boldsymbol{\xi}))
$$

Finally, we choose a linear interpolation which is a good compromise beetwen efficiency and speed.

\section{B. Optimization procedure}

We use an adaptive stochastic gradient descent procedure described in [21], given by:

$$
\boldsymbol{\mu}_{k+1}=\boldsymbol{\mu}_{k}-\gamma\left(t_{k}\right) \tilde{\mathbf{g}}_{k}, k=0,1, \ldots, K
$$

with $\boldsymbol{\mu}_{k}$ and $\boldsymbol{\mu}_{k+1}$ the transformation parameters at iteration $k$ and $k+1$, respectively. The search direction $\tilde{\mathbf{g}}_{k}$ is an approximation of the true derivative computed by central difference, and the gain factor $\gamma\left(t_{k}\right)$ is determined by a predefined decaying function of the iteration number $k$ :

$$
\begin{aligned}
\gamma\left(t_{k}\right) & =\frac{a}{\left(t_{k}+A\right)} \\
t_{k+1} & =\max \left[t_{k}+f\left(-\tilde{\mathbf{g}}_{k}^{\top} \tilde{\mathbf{g}}_{k-1}\right), 0\right]
\end{aligned}
$$

where $f$ denotes a sigmoid function. The originality of this algorithm comes from the automatic estimation of the free parameters, namely $a, A$ and $f$, before the registration starts.

Moreover, we choose a coarse to fine (or pyramidal) approach, which brings a speed-up in the registration. During rigid and affine registration procedures, the pyramidal approach consists in downsampling our images with a factor 2 at each level and performing the registration on the coarser level. When the optimum parameters are found, they are used to initialize the next level of the pyramid. During the non-rigid registration, the downsampling is done directly on the control grid of the B-Splines. We start with a coarse grid which is refined after the optimum is reached. This approach gives faster and better results, because it allows us to register global as well as local motion.

\section{Software implementation}

We use ITK (www.itk.org) implementations of $\mathrm{MI}_{\mathrm{G}}$ and $\mathrm{MI}_{\mathrm{BS}}$, namely MutualInformationImageToImageMetric and MattesMutualInformationImageToImageMetric. We also work with the elastiX toolbox [26] during the registration procedures (IV-B, IV-C), which allows the reproducibility of the results.

\section{EXPERIMENTS AND RESULTS}

The approximation of MI that we propose is evaluated and compared to both classic Parzen window approximations, solving increasingly difficult questions. First, we use these different approximations outside the registration context to evaluate their intrinsic behaviours. Then, we test the three similarity measures on two different medical image registration problems. We start with the rigid registration of multimodal skull volumes from the RIRE project. Next, we perform the non-rigid registration of CT lung volumes. For these registration tasks, the measures are used within a common registration scheme which allows for a direct comparison.

\section{A. Synthetic tests}

We first evaluate and compare the behaviors of the $\mathrm{MI}_{\mathrm{BS}}, \mathrm{MI}_{\mathrm{G}}$ and 3-EMI measures in the transformation space, by varying the translation parameters. The good behavior of a metric for registration purpose is characterized by its ability to measure the similarity between two images. A good metric is supposed to give a single global maximum that will make both images perfectly match. A 


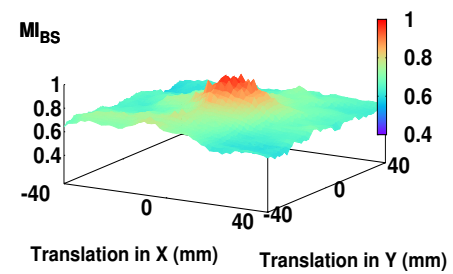

(a) $\mathrm{MI}_{\mathrm{BS}}, 50$ samples

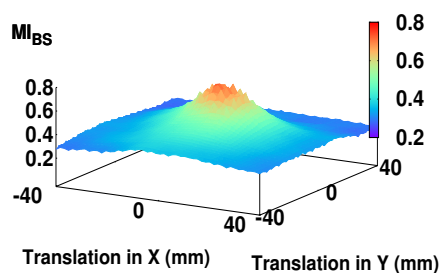

(d) $\mathrm{MI}_{\mathrm{BS}}, 500$ samples

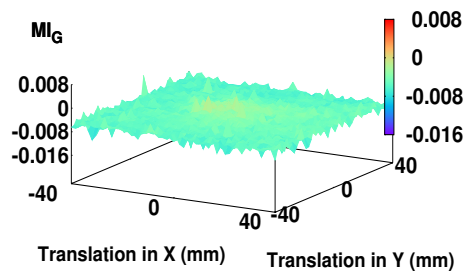

(b) $\mathrm{MI}_{\mathrm{G}}, 50$ samples

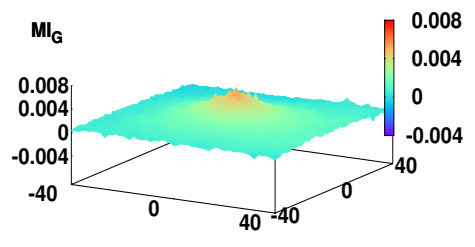

Translation in $X(\mathrm{~mm}) \quad$ Translation in $\mathrm{Y}(\mathrm{mm})$

(e) $\mathrm{MI}_{\mathrm{G}}, 500$ samples

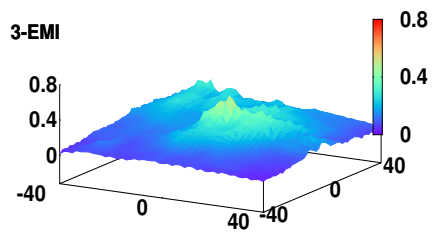

Translation in $X(\mathrm{~mm}) \quad$ Translation in $\mathrm{Y}(\mathrm{mm})$

(c) 3-EMI, 50 samples

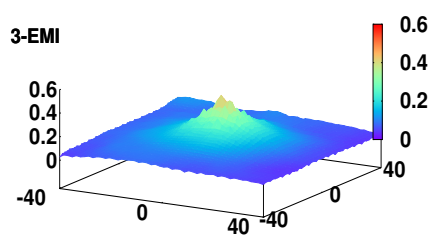

Translation in $X(\mathrm{~mm}) \quad$ Translation in $Y(\mathrm{~mm})$

(f) 3-EMI, 500 samples

Fig. 1. Behavior of the $\mathrm{MI}_{\mathrm{BS}}, \mathrm{MI}_{\mathrm{G}}$ and 3-EMI measures with a 10 degrees rotation, computed from 50 ((a), (b), (c)) or 500 ((e), (f), (g)) samples.

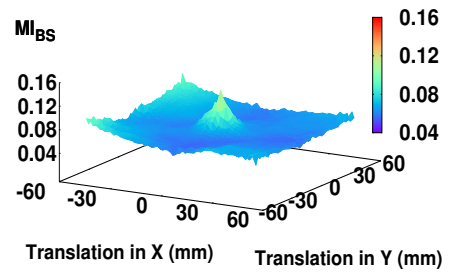

(a) $\mathrm{MI}_{\mathrm{BS}}$, standard deviation $=1$

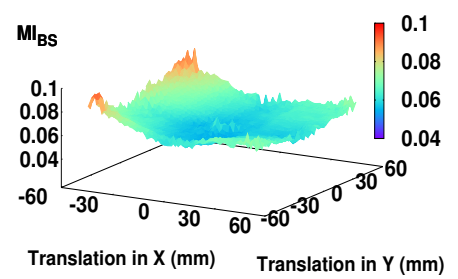

(d) $\mathrm{MI}_{\mathrm{BS}}$, standard deviation $=50$

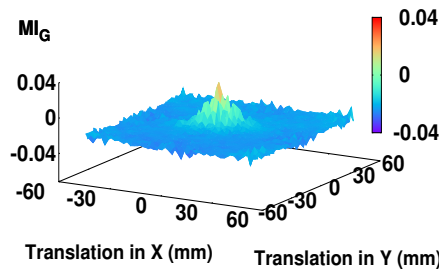

(b) $\mathrm{MI}_{\mathrm{G}}$, standard deviation $=1$

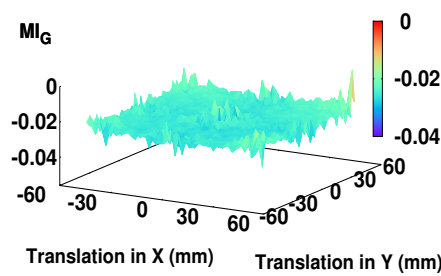

(e) $\mathrm{MI}_{\mathrm{G}}$, standard deviation $=50$

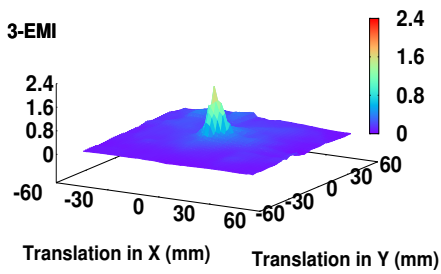

(c) 3-EMI, standard deviation $=1$

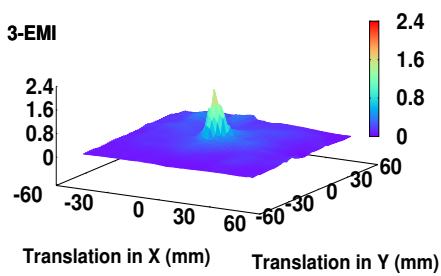

(f) 3-EMI, standard deviation $=50$

Fig. 2. Behavior of the $\mathrm{MI}_{\mathrm{BS}}, \mathrm{MI}_{\mathrm{G}}$ and 3-EMI measures computed from 50 samples using a moving image corrupted by an additive gaussian noise with standard deviation of 1 ((a) (b), (c)) or 50 ((e) (f), (g)).

low number of local maxima is also a criterion of good behavior. The quality of the registration procedure will directly depend on the behavior of the used measure. Indeed, the optimization methods can be very affected by the presence of a high number of local maxima. Hence, there is a real interest in studying the behavior of a measure before its use in registration, especially as a function of some influential parameters such as the variance of the omnipresent noise or the number of samples (pixels) used to compute the measure.

Our purpose is then to propose such an analysis by using real cardiac MSCT images. For each subject, we have access to a 3D volume of the heart taken every five percent of the cardiac cycle. The procedure consists first in randomly selecting a 2D slice among these $3 \mathrm{D}$ volumes that will be the reference image. Then, this image is translated along $x$ and $y$ directions between -40 and $+40 \mathrm{~mm}$ to obtain a moving image. For each translation value, we calculate the similarity measure between the reference and moving images. In order to get statistically significant results, we repeat the procedure on 100 randomly selected 2D images for each parameter configuration and each similarity measure, and we compute the average of all results. We also repeat these tests making the number of pixels used to compute the similarity measures vary. Indeed, this will have a great impact on the registration accuracy, and the overall computation time. Finally, the moving image was corrupted with additive Gaussian noise with different standard deviations. Complementary studies can be found in [22].

Figure 1 shows the behavior of the $\mathrm{MI}_{\mathrm{BS}}, \mathrm{MI}_{\mathrm{G}}$ and 3 -EMI measures for two numbers of samples: we use 50 pixels on the first raw of Fig. 1 and 500 on the second raw. In both cases, there is no noise and the rotation angle has been chosen equal to 10 degrees. The behavior of the $\mathrm{MI}_{\mathrm{BS}}$ and $\mathrm{MI}_{\mathrm{G}}$ measures is bad in terms of location of the global maximum and number of local maxima (Fig. 1(b)) when the number of pixels is low (50), which nevertheless is the recommended value in both the original article [3] and the ITK software. Our measure shows a better behavior. As expected, there is an improvement of the behavior of the three measures when 500 samples are used with a better localization of the global maximum on the 3-EMI measure.

Figure 2 displays the behavior of the $\mathrm{MI}_{\mathrm{BS}}, \mathrm{MI}_{\mathrm{G}}$ and 3 -EMI 
measures using an additive Gaussian noise with a standard deviation of 1 (first row) and 50 (second row), respectively, for 50 pixels and a zero rotation angle. This experiment shows the better behavior of our measure in the presence of a Gaussian noise, particularly when the noise becomes strong (second row of Fig.2). In such a case, the global maximum of the classical $\mathrm{MI}_{\mathrm{BS}}$ and $\mathrm{MI}_{\mathrm{G}}$ measures does not coincide with a perfect match between both images and a lot of spurious local maxima are present. On the contrary, our 3-EMI measure exhibits a global maximum at the good location without any local maximum in its neighborhood. The explanation is that a Gaussian variable has its cumulants of order strictly higher than 2 equal to 0 . Our measure, which is mainly composed of such cumulants, seems thus more robust with respect to the presence of an additive Gaussian noise.

\section{B. Rigid registration: RIRE database}

In this section, we consider the first nine subjects of the RIRE database [14]. The imaging modalities offered are CT, PET and MRI. Three types of MR images are considered: T1-weighted (denoted $\mathrm{T} 1$ ), T2-weighted (T2) and proton-density-weighted (PD). Moreover, these MR images have been corrected for static field inhomogeneity using [25], generating new rectified images (rectified). Finally, a goldstandard is provided for each registration, which allows for a quantitative evaluation of skull multimodal rigid registration. We perform a rigid registration of the nine patients, for each combination of imaging modalities, and for each considered similarity measure. We choose a common registration scheme for the different similarity measures, which allows for a fair comparison between the results. This scheme is composed of the adaptive stochastic gradient descent procedure [21] with automatic parameter estimation, coupled with a 5 levels pyramidal approach. The number of iterations is limited to 200 at each level of the pyramid, and we use 1000 pixels for the evaluation of the different similarity measures.

The results are given in table I and represent the mean registration error in $\mathrm{mm}$ for the first nine patients of the database, for each imaging couple and each similarity measure. The bold numbers represent the best result for each modality couple. We can see that our 3-EMI measure generally outperforms the classical $\mathrm{MI}_{\mathrm{BS}}$ and $\mathrm{MI}_{\mathrm{G}}$ ones. When it is not the case, our measure gives a result which is close to the best one. The superiority of 3-EMI is particularly true for PET/MRI registration tasks, which involve very different imaging modalities. Such a registration example is given in figure 3. We can also see from these tests the poor results obtained with $\mathrm{MI}_{\mathrm{G}}$ compared to both other measures. That's why we only consider the latter 2 metrics (namely 3-EMI and $\mathrm{MI}_{\mathrm{BS}}$ ) in the next evaluation section. We checked that the difference of results given in table I between the methods is statistically significant. This was confirmed by using a t-test (for 3-EMI, $\mathrm{MI}_{\mathrm{BS}}$, and $\mathrm{MI}_{\mathrm{G}}$ p-values are respectively $3.9 .10^{-212}, 2.0^{-165}$, and $1.8 .10^{-280}$ ). Note that we also checked using Kolmogorov's test that the difference of results was Gaussian in order to apply the t-test.

Finally, let's analyse the global numerical complexity involved by the repeated computations of each measure in the rigid registration task. $\mathrm{MI}_{\mathrm{G}}, \mathrm{MI}_{\mathrm{BS}}$ and 3 -EMI required $10^{9}, 1.024 * 10^{9}$ and $10^{6}$ flops, respectively.

\section{Non-rigid registration: EMPIRE10 Challenge}

Evaluation in the context of non-rigid registration is more ambitious because of the difficulty to use a reliable quantitative criterion. During MICCAI 2010 conference, the EMPIRE10 (Evaluation of Methods for Pulmonary Image Registration 2010) challenge [15]

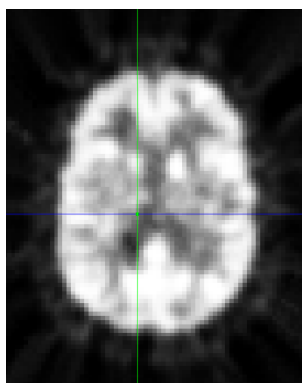

(a) PET

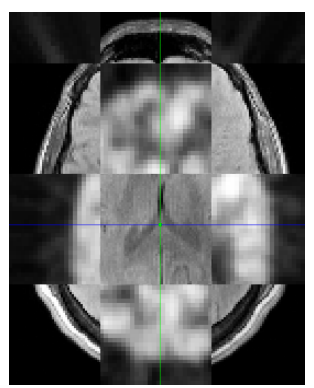

(c) Checkerboard before (d) registration

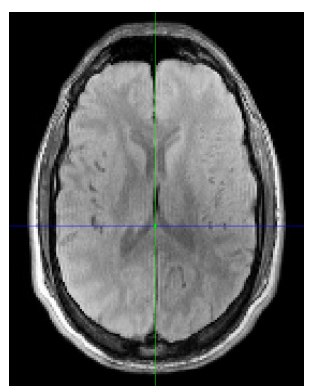

(b) MRI PD rectified

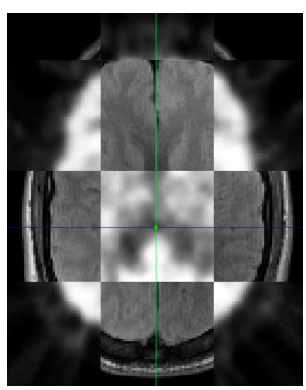

(d) Checkerboard after registration
Fig. 3. PET/MRI RIRE database registration example. (a) Original PET volume. (b) Original PD rectified MRI. (c) Checkerboard between reference and floating images before registration. (d) Checkerboard between reference and floating images after registration based on 3-EMI.

TABLE I

EVALUATION OF THE DIFFERENT METRICS IN THE CONTEXT OF RIGID REGISTRATION, USING THE RIRE DATABASE. THIS TABLE GIVES THE MEAN REGISTRATION ERROR (IN MM) OF THE FIRST NINE SUBJECTS OF THE DATABASE, FOR THE DIFFERENT IMAGING MODALITIES AND SIMILARITY MEASURES. THE BOLD NUMBERS REPRESENT THE BEST REGISTRATION FOR EACH IMAGE COUPLE.

\begin{tabular}{|c|c|c|c|c|}
\hline Modality 1 & Modality 2 & 3-EMI & $\mathrm{MI}_{\mathrm{BS}}$ & $\mathrm{MI}_{\mathrm{G}}$ \\
\hline $\mathrm{CT}$ & MRI_PD & 6.236 & 5.543 & 25.68 \\
\hline $\mathrm{CT}$ & MRI_PD_rectified & 5.03 & 4.808 & 26.747 \\
\hline $\mathrm{CT}$ & MRI_T1 & 5.459 & 6.686 & 36.282 \\
\hline $\mathrm{CT}$ & MRI_T1_rectified & 5.661 & 7.742 & 29.504 \\
\hline CT & MRI_T2 & 5.664 & 6.244 & 30.2 \\
\hline $\mathrm{CT}$ & MRI_T2_rectified & 5.777 & 5.349 & 33.976 \\
\hline PET & MRI_PD & 5.206 & 9.299 & 19.61 \\
\hline PET & MRI_PD_rectified & 5.122 & 5.55 & 20.473 \\
\hline PET & MRI_T1 & 5.378 & 9.692 & 28.218 \\
\hline PET & MRI_T1_rectified & 4.813 & 12.372 & 24.63 \\
\hline PET & MRI_T2 & 6.931 & 10.64 & 25.343 \\
\hline PET & MRI_T2_rectified & 7.156 & 8.092 & 28.018 \\
\hline \multicolumn{2}{|c|}{ Average } & 5.703 & 7.668 & 27.390 \\
\hline
\end{tabular}

[27] aimed at providing a platform for the evaluation of nonrigid registration algorithms. For this purpose, a database consisting of 30 subjects is used, and 2 CT volumes of the lungs taken at different times are available for each subject. Four criteria are used to assess the registration quality, but only two are presented hereafter: correspondence of annoted point pairs and analysis of singularities in the deformation field. We kept these two criteria that shown to be statistically significant using t-tests. The two others (lung boundary and fissure aligments) are not statistically significant (P-values of these criteria are respectively 0.9 and 0.4 ). . For each subject, one can obtain a score that has a different meaning regarding the criterion observed. For the correspondence of annoted point pairs criterion, the score represents the average distance between the considered points, 
while for the other criteria, the score represents a percentage of total checked points for which the considered criteria is not achieved. This database also offers the opportunity to submit the results online in order to challenge the other participants and to obtain the rank of proposed algorithms. More details regarding the evaluation and ranking procedures can be found on the website [15] and [27].

In this section, we compare the performance of 3-EMI and $\mathrm{MI}_{\mathrm{BS}}$ in the context of non-rigid registration using this database. Once again, we chose a common registration framework allowing for a fair comparison. The registration proceeds in 2 steps: first, an affine registration is performed as a preprocessing and second, the non-rigid registration takes place using the initial transformation found in the first stage as an initialization. In both procedures, an adaptive stochastic gradient descent optimizer with automatic parameter estimation is used [21]. For the affine registration, we apply a pyramidal approach with 5 levels, 1000 iterations per level and 2000 pixels to estimate the similarity measures. During the nonrigid registration based on B-Splines, we use a bi-pyramidal approach consisting in downsampling the images and gradually refining the BSplines control grid. We use 4 levels for each pyramid, the final spacing between the B-Spline control points is $20 \mathrm{~mm}$, we employ a maximum of 200 iterations per level and use 1000 pixels to estimate the measures.

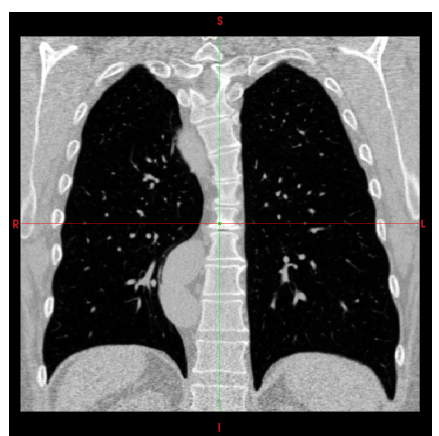

(a) Reference image

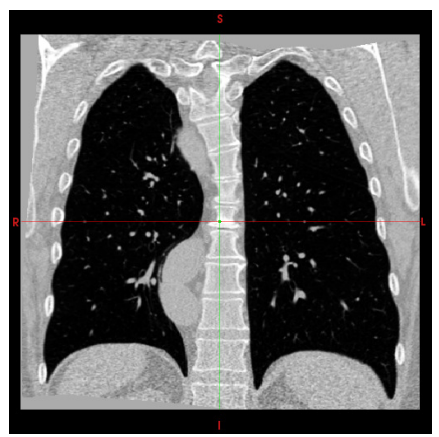

(c) Floating registered image

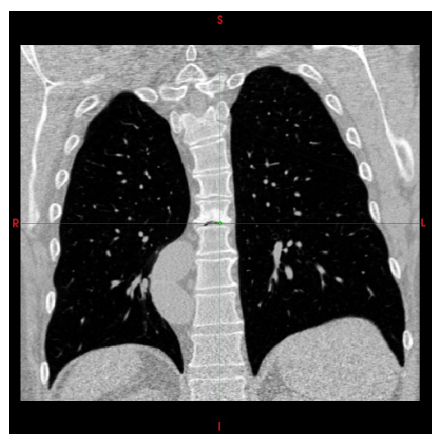

(b) Floating image

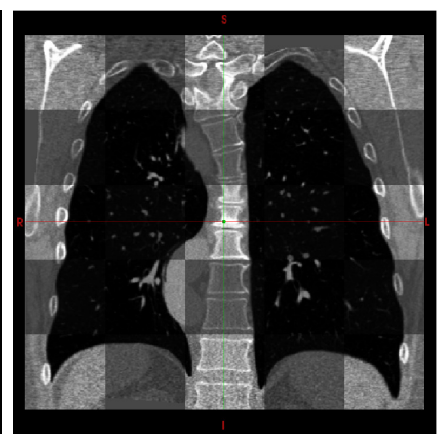

(d) Checkerboard after registration
Fig. 4. Example of monomodal non-rigid lung registration (subject 25, 3 EMI metric). (a) Reference image. (b) Floating image. (c) Floating registered image. (d) Checkerboard between (a) and (c). This example illustrates the good behaviour of the 3-EMI metric for non-rigid registration.

The global results for both similarity measures are given in table II. The ones for our 3-EMI metric are also available online at the address [15]. We can see that they are similar of slightly better than those obtained with $\mathrm{MI}_{\mathrm{BS}}$. This is particularly true for the evaluation based on landmarks (first criterion), where our metric performs better 28 times out of 30 . It is noteworthy that the latter results were shown to be statistically significant. If we detail these results, we can see that both methods give good results in general, but some of the subjects (1,
$7,14,18,20,21,28)$ present difficulties. They correspond to image couples that are very different one from the other. The consequence is that the overall results for both metrics can seem poor. Other teams competing in this challenge and using the same kind of framework (B-Spline non-rigid transformation model and MI similarity measure) usually add an energy term to prevent folding which can occur with these demanding subjects. Our aim was not to obtain the best possible results, but just to compare two similarity measures, in the context of non-rigid registration, in a common and simple possible framework which avoids other components of the scheme to interfer too much with the metrics. Hence these results show at once the feasibility of our measure for non-rigid registration, and the overall good behaviour of the registration framework. An illustrative example of the results we obtained is given in figure 4. This corresponds to the results for subject 25 registered using 3-EMI metric.

Finally, let's study the global numerical complexity involved by the repeated computations of each measure in this non-rigid registration task. $\mathrm{MI}_{\mathrm{BS}}$ and 3-EMI required $6.96 * 10^{9}$ and $11.6 * 10^{6}$ flops, respectively.

\section{CONCLUSION}

In this paper, we present a new similarity measure based on a third order approximation of MI using an Edgeworth expansion of PDF's and called 3-EMI. To begin, we show that this measure requires a few pixels to be estimated correctly, and is more robust to additive Gaussian noise than traditionnal MI estimators. We also demonstrate its ability to perform efficient rigid multimodal registration compared to the classical B-Spline Parzen window estimator of MI. Moreover, our measure exhibits a better robustness when the images to register have a very different intensity information, as in the case of PET/MRI registration. Finally, we establish using the EMPIRE database that it is also effective for non-rigid registration tasks, and this remains a challenge in medical image registration. This comes from the use of the cumulants, which brings a relevant statistical information about the images to register.

We also show that our measure gives these satisfactory results with furthermore a lower computational complexity per iteration than B-Spline estimator of MI. Moreover, in our experiments, the total number of iterations used by the optimizer was always the stopping criterion that was taken into account among others, regardless of the similarity measure used. So the overall registration procedure using our approximation is more efficient, regarding time consumption.

This study offers new perspectives regarding the use of MI in iconic medical image registration. First, the optimization approach uses an estimation of the gradient by finite difference causing estimation errors. However, our metric allows for an analytical calculation of the gradient. Such an exact calculation will improve the performance of the optimization algorithm. Secondly, the extension of our 3EMI similarity measure to higher orders will improve the quality of registration. Indeed, it will use a truncated edgeworth development to a higher order (eg 4 instead of 3), which will reduce the estimation error of the MI.

\section{ACKNOWLEDGMENT}

We would like to thank the Empire10 team for the medical testing data they provide to the scientific community. We also want to thank them for computing the results of our methods according to their evaluation criteria, and for making these results available online. 
TABLE II

EVALUATION OF 3-EMI AND MI BS METRICS IN THE CONTEXT OF MONOMODAL NON-RIGID LUNGS REGISTRATION, USING THE EMPIRE10 DATABASE. THE EVALUATION IS HELD REGARDING FOUR DIFFERENT CRITERIA, BUT ONLY THE TWO CRITERIA THAT ARE STATISTICALLY SIGNIFICANT ARE REPORTED IN THIS TABLE (LANDMARKS AND SINGULARITIES). FOR LANDMARKS CRITERION, THE SCORE REPRESENTS AN AVERAGE DISTANCE IN MM BETWEEN LANDMARKS. FOR THE OTHER CRITERION, THE SCORE IS A PERCENTAGE OF TOTAL CHECKED POINTS FOR WHICH A PENALTY OCCURED. A RANKING PROCESS BETWEEN THE DIFFERENT PARTICIPANTS IS ALSO USED. SEE [15] FOR MORE DETAILS REGARDING THE EVALUATION. BOLD NUMBERS REPRESENT THE BEST RESULT FOR EACH CRITERION AND EACH SUBJECT. COURTESY OF THE EMPIRE10 TEAM.

\begin{tabular}{|c|c|c|c|c|c|c|c|c|}
\hline & \multicolumn{4}{|c|}{ Landmarks } & \multicolumn{4}{|c|}{ Singularities } \\
\hline Scan & \multicolumn{2}{|c|}{ 3-EMI } & \multicolumn{2}{|c|}{$\mathrm{MI}_{\mathrm{BS}}$} & \multicolumn{2}{|c|}{ 3-EMI } & \multicolumn{2}{|c|}{$\mathrm{MI}_{\mathrm{BS}}$} \\
\hline Pair & Score & Rank & Scor & Rank & Score & Rank & Score & Rank \\
\hline 1 & 6.19 & 22.00 & 11.84 & 25.00 & 0.00 & 10.00 & 0.00 & 10.00 \\
\hline 2 & 0.99 & 23.00 & 1.96 & 25.00 & 0.00 & 11.50 & 0.00 & 11.50 \\
\hline 3 & 1.03 & 24.00 & 2.25 & 26.00 & 0.00 & 12.00 & 0.00 & 12.00 \\
\hline 4 & 1.85 & 17.00 & 3.10 & 23.00 & 0.00 & 11.50 & 0.00 & 11.50 \\
\hline 5 & 0.19 & 17.00 & 1.46 & 26.00 & 0.00 & 11.00 & 0.00 & 11.00 \\
\hline 6 & 0.96 & 24.00 & 1.82 & 27.00 & $\overline{0.00}$ & 13.00 & 0.00 & 13.00 \\
\hline 7 & 7.49 & 25.00 & 8.19 & 26.00 & 0.00 & 9.00 & 0.00 & 9.00 \\
\hline 8 & 3.14 & 22.00 & 5.01 & 26.00 & 0.00 & 11.50 & 0.00 & 11.50 \\
\hline 9 & 1.47 & 25.00 & 2.24 & 26.00 & 0.00 & 11.00 & 0.00 & 11.00 \\
\hline 10 & 3.62 & 19.00 & 4.38 & 21.00 & 0.00 & 11.00 & 0.00 & 11.00 \\
\hline 11 & 3.45 & 25.00 & 5.90 & 26.00 & 0.00 & 10.50 & 0.00 & 10.50 \\
\hline 12 & 0.65 & 21.00 & 2.58 & 26.00 & 0.00 & 12.50 & 0.00 & 12.50 \\
\hline 13 & 1.98 & 25.00 & 3.15 & 26.00 & 0.00 & 11.00 & 0.00 & 11.00 \\
\hline 14 & 13.10 & 25.00 & 13.52 & 26.00 & 0.00 & 7.50 & 0.00 & 7.50 \\
\hline 15 & 1.02 & 21.00 & 1.90 & 25.00 & 0.00 & 11.50 & 0.00 & 11.50 \\
\hline 16 & 2.08 & 23.00 & 5.34 & 26.00 & 0.00 & 12.00 & 0.00 & 12.00 \\
\hline 17 & 1.60 & 24.00 & 3.00 & 26.00 & $\overline{0.00}$ & 11.50 & 0.00 & 11.50 \\
\hline 18 & 9.16 & 25.00 & 9.81 & 26.00 & 0.00 & 9.00 & 0.00 & 9.00 \\
\hline 19 & $\overline{0.83}$ & 22.00 & 2.14 & 26.00 & $\overline{0.00}$ & 11.50 & 0.00 & 11.50 \\
\hline 20 & 12.90 & 25.00 & 7.16 & 22.00 & 0.00 & 10.00 & 0.00 & 10.00 \\
\hline 21 & 10.08 & 21.00 & 15.58 & 23.00 & 0.00 & 9.50 & 0.00 & 9.50 \\
\hline 22 & 1.95 & 23.00 & 4.23 & 26.00 & 0.00 & 11.00 & 0.00 & 11.00 \\
\hline 23 & 2.00 & 24.00 & 3.79 & 25.00 & $\overline{0.00}$ & 11.50 & 0.00 & 11.50 \\
\hline 24 & 1.18 & 14.00 & 2.85 & 26.00 & $\mathbf{0 . 0 0}$ & 11.00 & 0.00 & 11.00 \\
\hline 25 & 0.56 & 23.00 & 1.76 & 26.00 & 0.00 & 10.50 & 0.00 & 10.50 \\
\hline 26 & 1.34 & 24.00 & 2.14 & 27.00 & 0.00 & 12.50 & 0.00 & 12.50 \\
\hline 27 & 1.41 & 24.00 & 2.53 & 25.00 & 0.00 & 12.00 & 0.00 & 12.00 \\
\hline 28 & 9.85 & 25.00 & 7.19 & 24.00 & 0.00 & 10.50 & 0.00 & 10.50 \\
\hline 29 & 1.59 & 17.00 & 2.29 & 24.00 & 0.00 & 11.00 & 0.00 & 11.00 \\
\hline 30 & 1.09 & 24.00 & 1.87 & 25.00 & 0.00 & 12.00 & 0.00 & 12.00 \\
\hline Avg & 3.49 & 22.43 & 4.70 & 25.23 & 0.00 & 11.00 & 0.00 & 11.00 \\
\hline
\end{tabular}

APPENDiX A

\section{UNIVARIATE AND BIVARIATE HERMITE POLYNOMIALS}

The univariate Hermite polynomials are defined by:

$$
\phi_{x}(u) H_{k}(u)=(-1)^{k} \frac{d^{k} \phi_{x}(u)}{d u^{k}}
$$

where:

$$
\phi_{x}(u)=\frac{1}{\sqrt{2 \pi \operatorname{Var}(x)}} \mathrm{e}^{-(u-\mathrm{E}[x])^{2} /(2 \operatorname{Var}(x))}
$$

is the marginal normal distribution with $\operatorname{Var}(x)$ denoting the variance of $x$. The first seven univariate Hermite polynomials are given by:

$$
\begin{aligned}
& H_{0}(u)=1 \\
& H_{1}(u)=u \\
& H_{2}(u)=u^{2}-1 \\
& H_{3}(u)=u^{3}-3 u \\
& H_{4}(u)=u^{4}-6 u^{2}+3 \\
& H_{5}(u)=u^{5}-10 u^{3}+15 u \\
& H_{6}(u)=u^{6}-15 u^{4}+45 u^{2}-15
\end{aligned}
$$

the bidimensionnal normal distribution, where $\boldsymbol{\kappa}_{\boldsymbol{x}}=\mathrm{E}\left[\boldsymbol{x} \boldsymbol{x}^{\top}\right]-$ $\mathrm{E}[\boldsymbol{x}] \mathrm{E}\left[\boldsymbol{x}^{\mathrm{T}}\right]$ is the covariance matrix of $\boldsymbol{x}$ whose elements are denoted by $\kappa^{i, j}(\boldsymbol{x})$ and where $\boldsymbol{\kappa}_{\boldsymbol{x}}^{-1}$ is its inverse whose elements are written $\kappa_{i, j}(\boldsymbol{x})$. The first bivariate Hermite polynomial is given by:

$$
H_{i_{1}}(\boldsymbol{u})=\kappa_{i_{1}, 1}(\boldsymbol{x}) u^{(1)}+\kappa_{i_{1}, 2}(\boldsymbol{x}) u^{(2)}
$$

and the following recurrence relation:

$$
\partial_{i} H_{i_{1}, \ldots, i_{k}}(\boldsymbol{u})=\kappa_{i i_{1}}(\boldsymbol{x}) H_{i_{2}, \ldots, i_{k}}(\boldsymbol{u})[k]
$$

allows one to recover the other polynomials. In this last expression and the following, the notation $[k]$, introduced in [19] to simplify the

They enjoy the following orthogonality property: 
formulas, represents a sum of $k$ similar terms, determined by suitable permutations of the indices.

Their orthogonality property can be express as:

$$
\begin{gathered}
\oint_{\mathbb{R}^{2}} H_{i_{1}, \ldots, i_{k}}(\boldsymbol{u}) H_{j_{1}, \ldots, j_{\ell}}(\boldsymbol{u}) \phi_{\boldsymbol{x}}(\boldsymbol{u}) d \boldsymbol{u} \\
= \begin{cases}{[k !] \kappa_{i_{1}, j_{1}}(\boldsymbol{x}) \ldots \kappa_{i_{k}, j_{k}}(\boldsymbol{x})} & \text { if } \ell=k \\
0 & \text { otherwise }\end{cases}
\end{gathered}
$$

\section{APPENDIX B}

ZERO MEAN - UNIT VARIANCE BIVARIATE NORMAL DISTRIBUTION Considering $\boldsymbol{u}=\left(u_{1}, u_{2}\right)^{\top}$ and $\boldsymbol{\kappa}_{\boldsymbol{x}}=\left(\begin{array}{ll}1 & \rho \\ \rho & 1\end{array}\right)$ the covariance matrix of the zero mean unit variance random vector $\boldsymbol{x}$, we can write:

$$
\phi_{\boldsymbol{x}}(\boldsymbol{u})=\frac{1}{(2 \pi) \sqrt{1-\rho^{2}}} \exp \left(-\frac{1}{2\left(1-\rho^{2}\right)}\left(u_{1}^{2}+u_{2}^{2}-2 \rho u_{1} u_{2}\right)\right)
$$

the zero mean - unit variance bivariate normal distribution.

\section{APPENDIX C \\ THIRD ORDER EDGEWORTH-BASED APPROXIMATION OF JOINT ENTROPY}

We give here the mathematical developments needed to build the approximation of joint entropy for potentially correlated random variables. Approximation of marginal entropy can be found in a similar manner.

Including (6) in the definition of joint entropy (2), and using the second order Taylor expansion $(1+x) \log (1+x) \approx x+\frac{x^{2}}{2}$, we can write:

$$
\begin{aligned}
H(\boldsymbol{x}) & \approx-\oint_{\mathbb{R}^{2}} \phi_{\boldsymbol{x}}(\boldsymbol{u}) \log \left(\phi_{\boldsymbol{x}}(\boldsymbol{u})\right) d \boldsymbol{u} \\
& -\oint_{\mathbb{R}^{2}} \phi_{\boldsymbol{x}}(\boldsymbol{u}) \log \left(\phi_{\boldsymbol{x}}(\boldsymbol{u})\right) B_{\boldsymbol{x}}(\boldsymbol{u}) d \boldsymbol{u} \\
& -\oint_{\mathbb{R}^{2}} \phi_{\boldsymbol{x}}(\boldsymbol{u}) B_{\boldsymbol{x}}(\boldsymbol{u}) d \boldsymbol{u} \\
& -\frac{1}{2} \oint_{\mathbb{R}^{2}} \phi_{\boldsymbol{x}}(\boldsymbol{u})\left(B_{\boldsymbol{x}}(\boldsymbol{u})\right)^{2} d \boldsymbol{u}
\end{aligned}
$$

The first integral (25) is the entropy of a bivariate normal distribution given by $1+\log (2 \pi)+0.5 \log \left(1-\rho^{2}\right)$. After straightforward manipulations, and using i) the zero mean unit variance bivariate normal distribution (24) and ii) the orthogonality properties of bivariate Hermite polynomials (23), it can be shown that (26) and (27) are equal to zero. The last term (28) to compute can be rewritten:

$$
\begin{aligned}
& -\frac{1}{2} \oint_{\mathbb{R}^{2}} \phi_{\boldsymbol{x}}(\boldsymbol{u})\left(B_{\boldsymbol{x}}(\boldsymbol{u})\right)^{2} d \boldsymbol{u} \\
& =-\frac{1}{72} \oint_{\mathbb{R}^{2}} \phi_{\boldsymbol{x}}(\boldsymbol{u})\left(\left(\kappa^{1,1,1}\right)^{2} H_{111}(\boldsymbol{u})^{2}+9\left(\kappa^{1,1,2}\right)^{2} H_{112}(\boldsymbol{u})^{2}\right. \\
& +9\left(\kappa^{1,2,2}\right)^{2} H_{122}(\boldsymbol{u})^{2}+\left(\kappa^{2,2,2}\right)^{2} H_{222}(\boldsymbol{u})^{2} \\
& +6 \kappa^{1,1,1} \kappa^{1,1,2} H_{111}(\boldsymbol{u}) H_{112}(\boldsymbol{u})+6 \kappa^{1,1,1} \kappa^{1,2,2} H_{111}(\boldsymbol{u}) H_{122}(\boldsymbol{u}) \\
& +2 \kappa^{1,1,1} \kappa^{2,2,2} H_{111}(\boldsymbol{u}) H_{222}(\boldsymbol{u})+18 \kappa^{1,1,2} \kappa^{1,2,2} H_{112}(\boldsymbol{u}) H_{122}(\boldsymbol{u})
\end{aligned}
$$

$\left.+6 \kappa^{1,1,2} \kappa^{2,2,2} H_{112}(\boldsymbol{u}) H_{222}(\boldsymbol{u})+6 \kappa^{1,2,2} \kappa^{2,2,2} H_{122}(\boldsymbol{u}) H_{222}(\boldsymbol{u})\right) d \boldsymbol{u}$

To compute the ten integrals in (29), one needs once again to use the orthogonality properties of bivariate Hermite polynomials (23), with $\ell=k$. More precisely, we can write:

$$
\begin{aligned}
& \oint_{\mathbb{R}^{2}} \phi \boldsymbol{x}(\boldsymbol{u})\left(H_{1,1,1}(\boldsymbol{u})\right)^{2} d \boldsymbol{u}=\frac{6}{\left(1-\rho^{2}\right)^{3}} \\
& \oint_{\mathbb{R}^{2}} \phi \boldsymbol{x}(\boldsymbol{u})\left(H_{2,2,2}(\boldsymbol{u})\right)^{2} d \boldsymbol{u}=\frac{6}{\left(1-\rho^{2}\right)^{3}} \\
& \oint_{\mathbb{R}^{2}} \phi \boldsymbol{x}(\boldsymbol{u})\left(H_{1,1,2}(\boldsymbol{u})\right)^{2} d \boldsymbol{u}=\frac{2+4 \rho^{2}}{\left(1-\rho^{2}\right)^{3}} \\
& \oint_{\mathbb{R}^{2}} \phi \boldsymbol{x}(\boldsymbol{u})\left(H_{1,2,2}(\boldsymbol{u})\right)^{2} d \boldsymbol{u}=\frac{2+4 \rho^{2}}{\left(1-\rho^{2}\right)^{3}} \\
& \oint_{\mathbb{R}^{2}} \phi \boldsymbol{x}(\boldsymbol{u}) H_{1,1,1}(\boldsymbol{u}) H_{1,1,2}(\boldsymbol{u}) d \boldsymbol{u}=-\frac{6 \rho}{\left(1-\rho^{2}\right)^{3}} \\
& \oint_{\mathbb{R}^{2}} \phi \boldsymbol{x}(\boldsymbol{u}) H_{1,1,1}(\boldsymbol{u}) H_{1,2,2}(\boldsymbol{u}) d \boldsymbol{u}=\frac{6 \rho^{2}}{\left(1-\rho^{2}\right)^{3}} \\
& \oint_{\mathbb{R}^{2}} \phi \boldsymbol{x}(\boldsymbol{u}) H_{1,1,1}(\boldsymbol{u}) H_{2,2,2}(\boldsymbol{u}) d \boldsymbol{u}=-\frac{6 \rho^{3}}{\left(1-\rho^{2}\right)^{3}} \\
& \oint_{\mathbb{R}^{2}} \phi \boldsymbol{x}(\boldsymbol{u}) H_{1,1,2}(\boldsymbol{u}) H_{1,2,2}(\boldsymbol{u}) d \boldsymbol{u}=-\frac{4 \rho+2 \rho^{3}}{\left(1-\rho^{2}\right)^{3}} \\
& \oint_{\mathbb{R}^{2}} \phi \boldsymbol{x}(\boldsymbol{u}) H_{1,1,2}(\boldsymbol{u}) H_{2,2,2}(\boldsymbol{u}) d \boldsymbol{u}=\frac{6 \rho^{2}}{\left(1-\rho^{2}\right)^{3}} \\
& \oint_{\mathbb{R}^{2}} \phi \boldsymbol{x}(\boldsymbol{u}) H_{1,2,2}(\boldsymbol{u}) H_{2,2,2}(\boldsymbol{u}) d \boldsymbol{u}=-\frac{6 \rho}{\left(1-\rho^{2}\right)^{3}}
\end{aligned}
$$

which leads, after some operations, to:

$$
\begin{aligned}
& -\frac{1}{2} \oint_{\mathbb{R}^{2}} \phi_{\boldsymbol{x}}(\boldsymbol{u})\left(B_{\boldsymbol{x}}(\boldsymbol{u})\right)^{2} d \boldsymbol{u} \\
& =-\frac{1}{12\left(1-\rho^{2}\right)^{3}}\left[\kappa^{1,1,1^{2}}+\kappa^{2,2,2^{2}}+3 \kappa^{1,1,2^{2}}+3 \kappa^{1,2,2^{2}}\right. \\
& -6 \rho\left(\kappa^{1,1,1} \kappa^{1,1,2}+\kappa^{1,2,2} \kappa^{2,2,2}+2 \kappa^{1,1,2} \kappa^{1,2,2}\right) \\
& +6 \rho^{2}\left(\kappa^{1,1,2^{2}}+\kappa^{1,2,2^{2}}+\kappa^{1,1,1} \kappa^{1,2,2}+\kappa^{1,1,2} \kappa^{2,2,2}\right) \\
& \left.-2 \rho^{3}\left(\kappa^{1,1,1} \kappa^{2,2,2}+3 \kappa^{1,1,2} \kappa^{1,2,2}\right)\right]
\end{aligned}
$$

Reordering the different terms of the approximation leads to (9).

\section{REFERENCES}

[1] Brown, L.G., A survey of image registration techniques,ACM Computer Survey, 1992, vol.24, $\mathrm{n}^{\circ} 4$

[2] Zitova, B. and Flusser, J., Image registration methods: a survey,Image and Vision Computing, 2003, vol.21, pp.977-1000.

[3] Viola, P.A. and Wells, W., Alignement by maximization of mutual information, International Conference on Computer Vision, IEEE Computer Society Press, Los Alamitos, CA, 1995, pp.16-23.

[4] Collignon, A. and Vandermulen, D. and Suetens, P. and Marchal, G., 3D Multi-Modality Medical Image Registration Using Feature Space Clustering,CVRMed '95: Proceedings of the First International Conference on Computer Vision, Virtual Reality and Robotics in Medicine, 1995, vol.905, pp.195-204.

(295) Maintz, J.B.A. and Viergever, M.A., A survey of medical image registration, Medical Image Analysis, 1998, vol.2, pp.1-36, $\mathrm{n}^{\circ} 1$.

[6] Pluim, J.P.W and Maintz, J.B.A. and Viergever, M.A., Mutual information based registration of medical images: a survey,IEEE Transactions on Medical Imaging, 2003, vol.22, pp.986-1004.

[7] Moddemeijer, R., On estimation of entropy and mutual information of continuous distributions,Signal Processing, 1989, vol.16, pp.233-248. 
[8] Mars, N.J.I. Van Arragon, G.W., Time delay estimation in non-linear systems using average amount of mutual information analysis, Signal Processing, 1982, vol.4, pp.139-153.

[9] Moon, Young-Il and Rajagopalan, Balaji and Lall, Upmanu, Estimation of mutual information using kernel density estimators, Physical Review E, 1995, vol.52, pp.2318-2321, no3.

[10] Comon, P., Independent Component Analysis, a new concept ?,Signal Processing, Elsevier, 1994, vol.36, pp.287-314, nº3.

[11] Thévenaz, P. and Unser, M., Spline Pyramids for Inter-Modal Image Registration Using Mutual Information,Proceedings of the SPIE Conference on Mathematical Imaging: Wavelet Applications in Signal and Image Processing V, 1997, vol.3169, pp.236-247, San Diego CA, USA.

[12] Mattes, D. and Haynor, D.R. and Vesselle, H. and Lewellen, T.K. and Eubank, W., PET-CT Image Registration in the Chest Using Free-form Deformations., IEEE Transactions on Medical Imaging, 2003, vol.22, pp. $120-128, \mathrm{n}^{\mathrm{o}} 1$.

[13] Rubeaux, M. and Nunes, J.C. and Albera, L. and Garreau, M., Edgeworth-based approximation of Mutual Information for medical image registration, IEEE Image Processing Tools and Applications, pp.195-200, 2010.

[14] West, J. and al., Comparison and Evaluation of Retrospective Intermodality Image Registration Techniques, Journal of Computer Assisted Tomography, 1997, vol.21, pp.554-566, nº4.

[15] Evaluation of Methods for Pulmonary Image Registration (EMPIRE), 2010, http://empire10.isi.uu.nl/index.php.

[16] Shannon, C.E., A mathematical theory of communication, Bell Syst. Tech. J., 1948, vol.27, pp.379-423/623-656.

[17] Studholme, C. and Hill, D.L.G. and Hawkes, D.J., An overlap invariant entropy measure of 3D medical image alignment, Pattern Recognition, 1999, vol.32, pp.71-86, nº 1 .

[18] Van Hulle, Marc M., Edgeworth Approximation of Multivariate Differential Entropy, Neural Computation, 2005, vol.17, pp.1903-1910, nº9.

[19] Asymptotic techniques for use in statistics,Chapman and Hall, 1989, Barndorff-Nielsen, O.E. and Cox, D.R., (Monographs on statistics and applied probability).

[20] Amari, S. and Cichocki, A. and Yang, H. H., A new learning algorithm for blind signal separation, Advances in Neural Information Processing Systems, The MIT Press, 1996, vol.8, pp.757-763.

[21] Klein, S. and Pluim, J.P.W. and Staring, M. and Viergever,M.A., Adaptive stochastic gradient descent optimisation for image registration, International Journal of Computer Vision, 2009, vol.81, pp.227-239, nº3.

[22] Rubeaux, M., Approximation de l'Information Mutuelle basée sur le développement d'Edgeworth : application au recalage d'images médicales.,Université de Rennes 1, PhD thesis, 2011.

[23] Gholipour, A. and Kehtarnavaz, N.D., Computationally efficient mutual information estimation for non-rigid image registration., IEEE International Conference on Image Processing, 2008, pp.1792-1795.

[24] Rueckert, D. and Sonoda, L.I. and Hayes, C. and Hill, D.L.G. and Leach, M.O. and Hawkes, D.J., Nonrigid, registration using Free-Form Deformations: Application to breast MR images, IEEE Transactions on Medical Imaging, 1999, vol.18, pp.712-721, no8.

[25] Chang, H. and Fitzpatrick, J. M., A technique for accurate magnetic resonance imaging in the presence of field inhomogeneities, IEEE Transactions on Medical Imaging, 1992, vol.11, pp.319-329, n³.

[26] Klein, S. and Staring, M. and Murphy, K. and Viergever, M.A. and Pluim, J.P.W., elastix: a toolbox for intensity-based medical image registration, IEEE Transactions on Medical Imaging, 2010, vol.29, pp.196-205, $\mathrm{n}^{\mathrm{o}} 1$.

[27] Murphy, K. et al., Evaluation of Registration Methods on Thoracic CT: The EMPIRE10 Challenge, IEEE Transactions on Medical Imaging, 2011, vol.30, pp. 1901-1919, nº 11 . 